\title{
Generalized closing theorems
}

\author{
V.Yu. Protasov* \\ Vladimir Yu. Protasov received his Ph.D. from Moscow State University (MSU) in \\ 1999. In the following years he was a member of the Institute of Advanced Study in \\ Princeton (USA) and a post-doc at the Erasmus University in Rotterdam (The Nether- \\ lands). In 2006 he received his habilitation from the Steklov Institute in St. Petersburg. \\ Presently, he is a professor at MSU. His research interests comprise functional analy- \\ sis, operator and matrix theory, and optimization.
}

\section{Introduction}

Closing theorems, or theorems of Poncelet type, are considered to be one of the most fascinating geometric facts. Various approaches to their proofs as well as applications to problems of elementary geometry, theory of algebraic curves, differential equations, billiards, elliptic integrals, etc., have been studied in many works (see, for example, [1, $2,4,6,8,10,11,14]$, and references therein). One can spot the four best known closing theorems: Poncelet, Steiner, zigzag, and Emch theorems. We do not mention some other results, such as, for instance, the Ponzag theorem, that are actually reformulations of one of these four theorems.

This paper consists of two parts. First, in Section 3 we derive a general closing theorem for families of Euclidean spheres in $\mathbb{R}^{d}$. Then, in Sections 4-6, we observe some of its

* The research is supported by the RFBR grants No 10-01-00293 and No 11-01-00329.

Den meisten Lesern dürften die klassischen Schließungssätze der Elementargeometrie von Poncelet, Steiner und Emch sowie das Zigzag-Theorem bekannt sein. Beispielsweise zeigt der Schließungssatz von Poncelet ausgehend von einem $n$-Eck $(n>2)$, das gleichzeitig einem Kegelschnitt $C$ umschrieben und einem anderen Kegelschnitt $D$ einbeschrieben werden kann, dass es noch unendlich viele weitere $n$-Ecke mit dieser Eigenschaft gibt. In der vorliegenden Arbeit beweist der Autor einen allgemeinen Schließungssatz für Sphären im $\mathbb{R}^{d}$, aus dem die oben genannten klassischen SchlieBungssätze durch geeignete Spezialisierung unmittelbar gefolgert werden können. 
corollaries. One of them, Theorem 2, gives a general closing principle for spheres in the space $\mathbb{R}^{3}$. The four classical theorems of Poncelet type are its direct corollaries (Section 5). Another one, Theorem 3, extends the Emch closing theorem to spheres in the Euclidean space $\mathbb{R}^{d}$. In the second part of the paper, in Sections 7 and 8, we focus on the elementary proof of Emch's theorem on circular series. This theorem is the most general one among these four classical results. The Poncelet theorem in case of two circles, the planar version of the zigzag theorem, and the Steiner theorem are actually its special cases. This is shown in Section 7. Therefore it would be interesting to obtain an autonomous (not relying on the Poncelet theorem) proof of the Emch theorem using only elementary geometrical tools. Such a proof is given in Section 7. For the sake of simplicity we restrict ourselves to the case, when the three circles are embedded to each other. That proof is based on two auxiliary geometric results, Theorem 4 on four circles touching two concentric circles, and Proposition 1 on two chains of circles inscribed in an annulus, which may be of independent interest. Finally, in Section 8 we apply this technique to derive a generalization of the Emch theorem to pencils of circles.

\section{Four classical closing theorems}

In this section we recall the statements of the four famous Poncelet type theorems. To formulate them in a unique way it is convenient to introduce the notion of general closing property for families of circles. This notion will be also used in the next sections, when we generalize Poncelet type theorems to the space $\mathbb{R}^{d}$. Suppose a circle $\delta$ and a family of circles $\mathcal{M}$ are given on the plane $\mathbb{R}^{2}$. Straight lines and points are also considered as circles. We call a point $z \in \mathbb{R}^{d}$ singular for the family $\mathcal{M}$ if there are more than two circles from $\mathcal{M}$ passing through $z$. Assume that the two conditions are satisfied:

(a) The circle $\delta$ does not contain singular points for $\mathcal{M}$.

(b) $\delta \notin \mathcal{M}$.

Let us now consider the following process. Take an arbitrary point $D_{1}$ on $\delta$ and draw a circle $v_{1} \in \mathcal{M}$ through it (we suppose that such a circle exists; if there are two ones, then we take any of them). Let $D_{2}$ be the second point of intersection of $v_{1}$ and $\delta$ (in case of tangency we set $D_{2}=D_{1}$ ). Draw a circle $v_{2} \in \mathcal{M}$ through $D_{2}$ different from $v_{1}$ (if it does not exist, then we set $v_{2}=v_{1}$ ). Then we denote by $D_{3}$ the second point of intersection of $v_{2}$ and $\delta$, etc. We obtain a series of circles $\left\{v_{k}\right\}_{k=1}^{\infty}$. The process has period $n$ if $v_{n+1}=v_{1}$ or, which is the same, $D_{n+1}=D_{1}$.

Definition 1. A family of circles $\mathcal{M}$ is said to possess the closing property on a circle $\delta$ if it satisfies conditions (a), (b) and the following condition:

If for some initial point $D_{1}$ the process has period $n \geq 3$ and all the points $D_{1}, \ldots, D_{n}$ are distinct, then it has the same period for any point $D_{1} \in \delta$ that belongs to a circle from $\mathcal{M}$.

Now we are giving the statements of the four classical closing theorems.

Theorem A. [Poncelet [12]] For any quadric $\alpha$ and a circle $\delta$ on the plane the set of lines touching $\alpha$ possesses the closing property on $\delta$. 
As usual we call quadric in $\mathbb{R}^{d}$ a set of points $x \in \mathbb{R}^{d}$ such that $(x, A x)+(b, x)+c=0$, where $A$ is a self-adjoint operator, $b \in \mathbb{R}^{d}, c \in \mathbb{R}$, and $(\cdot, \cdot)$ denotes the standard inner product in $\mathbb{R}^{d}$. We deal with real nonempty quadrics only. A quadric in $\mathbb{R}^{2}$ is called plane. If the quadric $\alpha$ in Theorem $\mathrm{A}$ is degenerate (a pair of lines, a single line, or a point), then one replaces the tangents by parallel lines (in case of a line and of a pair of lines) or, in case of a point, by lines passing through that point. The Poncelet theorem is usually formulated for two quadrics $\alpha$ and $\delta$. Nevertheless, it can always be assumed that $\delta$ is a circle. Indeed, if the quadric $\delta$ is nondegenerate, then one can map it to a circle by a suitable stereographic projection. Therefore, in this case the Poncelet theorem for two quadrics follows from Theorem A. If $\delta$ is degenerate, then the Poncelet theorem is trivial, and the reader will easily prove it.

To illustrate the Poncelet theorem, consider the case, when $\alpha$ and $\delta$ are both circles, and $\alpha$ lies inside $\delta$. Take a point $D_{1} \in \delta$ and draw a line tangent to $\alpha$, which intersects the circle $\delta$ for the second time at a point $D_{2}$. There are two tangents to the circle $\alpha$ passing through $D_{2}$. One of them is $D_{2} D_{1}$. Draw the second one $D_{2} D_{3}$ (the point $D_{3}$ lies on $\delta$ ). Then we draw the next tangent $D_{3} D_{4}$ not coinciding with the previous one, etc. The Poncelet theorem says that if this process cycles after $n$ steps, i.e., $D_{n+1}=D_{1}$ (Fig. 1), then it

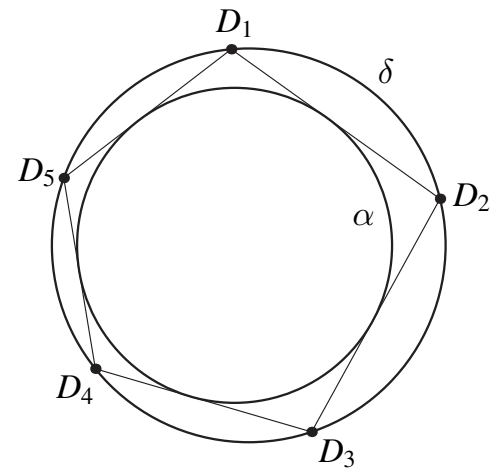

Fig. 1 Poncelet theorem

will cycle for any choice of the initial point $D_{1}$ with the same number of steps $n$. Thus, if there is an $n$-gon inscribed in the circle $\delta$ and circumscribed around the circle $\alpha$, then there are infinitely many such $n$-gons. Moreover, any point of the circle $\delta$ is a vertex of one of those inscribed-circumscribed $n$-gons. A beautiful proof of this version of Theorem A using measure theory was derived by Jacobi and Bertrand (see, for instance, [14]). Proofs of Theorem A involving the theory of projective quadrics see in $[3,11]$. Other proofs based on various ideas can be found in $[1,2,8,10]$. None of them is elementary.

To formulate the next classical closing theorem in its most general form we use the notion of index of tangency. The tangency of two circles is called interior if one of the circles lies inside the other. Suppose $\alpha_{0}, \alpha_{1}$ are circles on the plane; then for an arbitrary circle $\beta$ touching both $\alpha_{0}$ and $\alpha_{1}$ the index of tangency is 0 if there is an even number of interior tangencies among the two ones: $\beta$ with $\alpha_{0}$ and $\beta$ with $\alpha_{1}$. If this number is odd, then the index is 1 . This notion is naturally extended to the case, when some of the circles $\alpha_{j}$ 
become straight lines (the index depends on the orientation of the line). For given $i=0,1$ we denote by $\mathcal{M}_{i}$ the family of circles touching $\alpha_{0}$ and $\alpha_{1}$ with index $i$. If either $\alpha_{0}$ or $\alpha_{1}$ becomes a point, then $\mathcal{M}_{0}=\mathcal{M}_{1}$. The same notation will be used in the next sections for families of spheres in $\mathbb{R}^{3}$ touching two given spheres.

Given two circles $\alpha_{0}$ and $\alpha_{1}$ on the plane, $\alpha_{0}$ inside $\alpha_{1}$. In this case the family $\mathcal{M}_{1}$ consists of circles inscribed in the annulus formed by the circles $\alpha_{0}$ and $\alpha_{1}$. The process of Steiner produces a series of circles $\left\{v_{k}\right\}_{k \in \mathbb{N}} \subset \mathcal{M}_{1}$ as follows: $v_{1} \in \mathcal{M}_{1}$ is arbitrary, for any $k \in \mathbb{N}$ the circle $v_{k+1}$ touches $v_{k}$ and is different from $v_{k-1}$ if $k \geq 2$. The process has period $n \geq 3$ if $v_{n+1}=v_{1}$.

Theorem B. [Steiner] If the process of Steiner is periodic for some initial circle $v_{1}$, then it has the same period for any $v_{1} \in \mathcal{M}_{1}$.

Thus, if there is a closed chain of $n$ touching circles inscribed in the annulus between $\alpha_{0}$ and $\alpha_{1}$ (Fig. 2), then there are infinitely many such chains, and any circle inscribed in the annulus can be the first circle of a chain. This construction is sometimes called Steiner's necklace, or even Steiner's telephone dialer. In contrast to other closing theorems Theorem B has several elementary proofs. The most known one is by inversion: if one applies a suitable inversion taking $\alpha_{0}$ and $\alpha_{1}$ to a pair of concentric circles, then the statement becomes obvious. However, none of those elementary proofs can be extended to the other Poncelet type theorems.

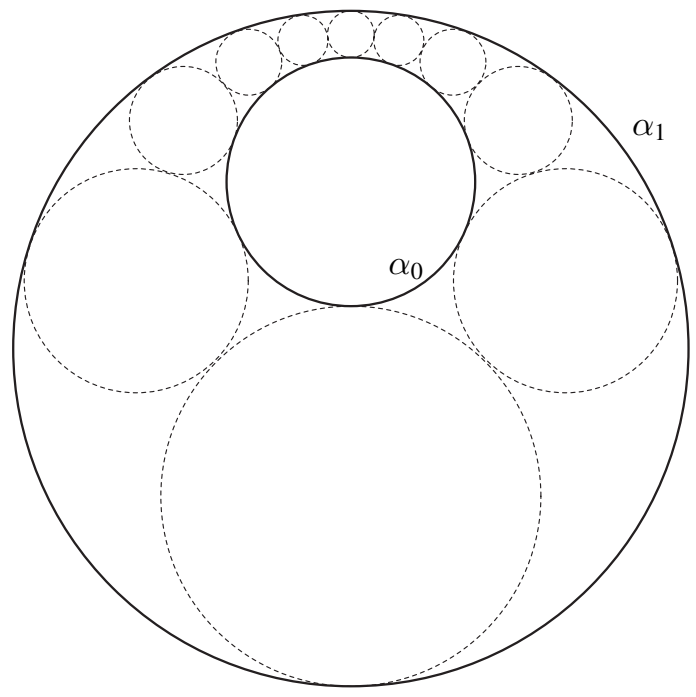

Fig. 2 Steiner theorem

The third one is the zigzag theorem. It also deals with two circles, but this time the circles are not necessarily on one plane, they may have arbitrary positions in the space. Given a number $\rho>0$ and two circles $s$ and $\delta$ in the space $\mathbb{R}^{3}$. Assume this pair of circles satisfies the following condition: 
(c) The orthogonal projection of any of these circles onto the two-dimensional plane containing the other circle does not pass through its center.

So, if one takes either of these circles and erects a perpendicular to its plane at the center of that circle, then it does not meet the other circle.

Take an arbitrary point $D_{1} \in \delta$. If the sphere of radius $\rho$ centered at $D_{1}$ intersects $s$, then we take any of two points of intersection and call it $S_{1}$. Then we take a point $D_{2} \in \delta$ such that $D_{2} S_{1}=\rho$ and $D_{2} \neq D_{1}$ (if it does not exist, then $D_{2}=D_{1}$ ). Further, the point $S_{2} \in s$ is such that $S_{2} D_{2}=\rho, S_{2} \neq S_{1}$ (if such a point does not exist, then we set $S_{2}=S_{1}$ ), and so on. The zigzag process produces the sequences $\left\{D_{k}\right\}$ and $\left\{S_{k}\right\}$ for a given initial segment $D_{1} S_{1}=\rho$. Zigzag has period $n$ if $D_{n+1}=D_{1}$.

Theorem C. [zigzag] If the zigzag has period $n \geq 3$ for some initial point $D_{1} \in \delta$ and all the intermediate points are distinct, then it has the same period for any point $D_{1} \in \delta$, from which one can make the first step.

The zigzag process can be interpreted as jumps of a flea from one circle to another with the same length of the jump $\rho$ (Fig. 3). If after $2 n$ jumps the flea arrives at the starting point $D_{1}$, then it will happen for any starting point on the circle $\delta$. In other words, if there is a $2 n$-gon, whose even vertices lie on the circle $\delta$, odd vertices lie on the circle s, and all sides have the same length $\rho$, then there are infinitely many such $2 n$-gons. Moreover, any point of $\delta$ can be a vertex of such a $2 n$-gon.

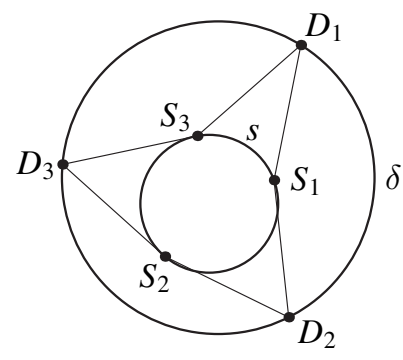

Fig. 3 Zigzag theorem

Theorem C originates in [5]. Its proofs based on various ideas, highly non-elementary, can be found in $[1,4]$. The equivalence of the zigzag theorem and the Poncelet theorem was established in [9].

Now we turn to the fourth closing theorem. We are going to see that this theorem is, in a sense, the strongest one: the three others easily follow from it. In the statement we again use the families of circles $\mathcal{M}_{0}$ and $\mathcal{M}_{1}$ defined above.

Theorem D. [Emch [7]] There are circles $\alpha_{0}, \alpha_{1}$, and $\delta$ on the plane, each of them may become a point. Then for any $i \in\{0,1\}$ the family $\mathcal{M}_{i}$ corresponding to the pair of circles $\alpha_{0}$, $\alpha_{1}$ possesses the closing property on $\delta$, provided $\delta \notin \mathcal{M}_{i}$.

Fig. 4 illustrates Theorem $\mathrm{D}$ in case, when the circle $\delta$ lies between $\alpha_{0}$ and $\alpha_{1}$. If there is a closed chain of $n$ circles inscribed in the annulus formed by $\alpha_{0}$ and $\alpha_{1}$, such that each pair 


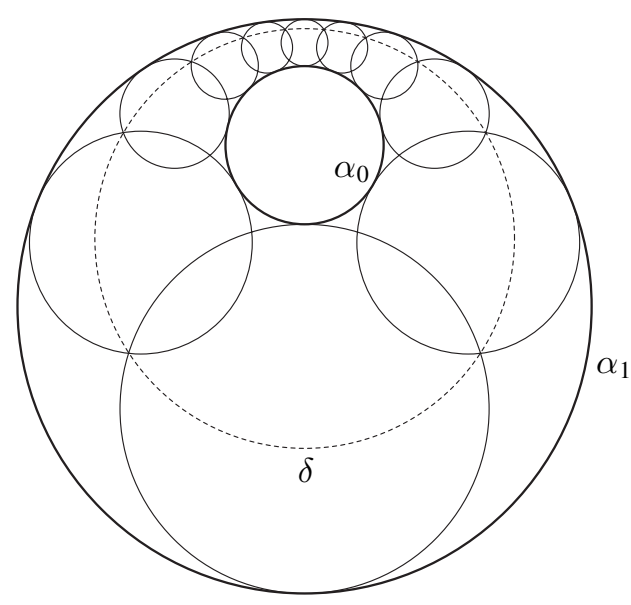

Fig. 4 Emch theorem

on neighboring circles meets on $\delta$, then there are infinitely many such chains. Moreover, any circle inscribed in the annulus can be the first circle of a chain.

A proof of Theorem D can be found in [1]. In [13] this theorem was derived from the Poncelet theorem by elementary geometric tools.

\section{General closing principle}

We are going to establish a fundamental theorem that implies not only the classical Theorems A-D, but also their multidimensional generalizations obtained in the next sections. This theorem is formulated in the space $\mathbb{R}^{d}$ for series of Euclidean spheres. Let us start with introducing some notation. We denote by $S(z, r)=\left\{x \in \mathbb{R}^{d},|x-z|=r\right\}$ a Euclidean sphere in $\mathbb{R}^{d}$ of radius $r$ centered at $z$; by $P(n, c)=\left\{x \in \mathbb{R}^{d},(n, x)=c\right\}$ we denote a hyperplane with a direction vector $n,|n|=1$, and $c \in \mathbb{R}$. A sequence of spheres $S\left(z_{k}, r_{k}\right)$, $k \in \mathbb{N}$, converges to the plane $P(n, c)$ if $r_{k} \rightarrow \infty, z_{k} /\left|z_{k}\right| \rightarrow n$ and $\left(\left|z_{k}\right|^{2}-r_{k}^{2}\right) / 2\left|z_{k}\right| \rightarrow c$ as $k \rightarrow \infty$. By spheres we also mean points (when $r=0$ ) and planes, unless the opposite is stated (for instance, when the radius is given). In particular, $S(z, r)$ denotes a sphere or a point (when $r=0$ ), but not a plane.

Let us now define the closing property for families of spheres in $\mathbb{R}^{d}$. Suppose a circle $\delta$ and a family of spheres $\mathcal{M}$ are given in the space $\mathbb{R}^{d}$. We call a point $z \in \mathbb{R}^{d}$ singular for the family $\mathcal{M}$ if there are more than two spheres from $\mathcal{M}$ passing through $z$. The two following conditions extend conditions (a) and (b) (Section 2) from circles to spheres:

$\left(\mathrm{a}^{\prime}\right)$ Circle $\delta$ does not contain singular points for $\mathcal{M}$.

$\left(\mathrm{b}^{\prime}\right)$ There is no sphere in $\mathcal{M}$ containing $\delta$.

Consider now the same process as in Section 2 for spheres. For an arbitrary point $D_{1}$ on the circle $\delta$ we draw a sphere $v_{1} \in \mathcal{M}$ through it (we suppose that such a sphere exists; if there are two ones, then we take any of them), and denote by $D_{2}$ the second point of intersection 
of $v_{1}$ and $\delta$ (in case of tangency $D_{2}=D_{1}$ ). Draw a sphere $v_{2} \in \mathcal{M}$ through $D_{2}$ different from $v_{1}$ (if it does not exist, then we set $v_{2}=v_{1}$ ), and denote by $D_{3}$ the second point of its intersection with $\delta$, etc. We obtain a series of spheres $\left\{v_{k}\right\}_{k=1}^{\infty}$. The process has period $n$ if $v_{n+1}=v_{1}$ or, which is the same, $D_{n+1}=D_{1}$.

Definition 2. A family of spheres $\mathcal{M}$ is said to possess the closing property on a circle $\delta$ if it satisfies conditions $\left(a^{\prime}\right),\left(b^{\prime}\right)$ and the following condition:

If for some initial point $D_{1}$ the process has period $n \geq 3$ and all the points $D_{1}, \ldots, D_{n}$ are distinct, then it has the same period for any point $D_{1} \in \delta$ that belongs to a sphere from $\mathcal{M}$.

The main result of this section gives sufficient conditions for a family of spheres to possess the closing property on any circle. Suppose we are given a set $\Gamma \subset \mathbb{R}^{d}$, which is either a plane quadric or a subset of a straight line. For arbitrary $a \in \mathbb{R}^{d}$ and $b \in \mathbb{R}$ consider the set of spheres $\left\{S(z, r) \subset \mathbb{R}^{d}\right\}$ defined by the following relations:

$$
r^{2}=|z-a|^{2}+b, \quad z \in \Gamma \text {. }
$$

So, this family consists of all spheres $S(z, r)$ such that $z \in \Gamma$ and $|z-a|^{2}+b \geq 0$, in which case $r=\sqrt{|z-a|^{2}+b}$. If the set $\Gamma$ is unbounded, then we add to this family one or two limit planes: if $\Gamma$ is a hyperbola or a pair of lines, then the two planes $P\left(n_{k}, c_{k}\right)$, $k=0,1$, are added, where $n_{k}$ are the direction vectors of the lines or of the asymptotes of the hyperbola, $c_{k}=\left(n_{k}, a\right)$; if $\Gamma$ is a subset of a line, then one plane $P(n, c)$ is added, where $n$ is the direction vector of the line, $c=(n, a)$.

Theorem 1. Family (1) possesses the closing property on any circle $\delta \subset \mathbb{R}^{d}$ that does not contain singular points for family (1) and does not lie on its spheres.

Proof. First, we reduce the theorem to the planar case, i.e., to $d=2$. Then we show that all circles of family (1) touch a suitable quadric $\alpha$, after which the theorem will follow from Theorem A.

Thus, let us reduce the theorem to the case $d=2$. We consider only spheres of family (1), the same results for planes, if they exist, will follow from the limit passage. Without loss of generality it may be assumed that the origin is located on the two-dimensional affine plane $K$ containing the circle $\delta$. If some sphere $S(z, r)$ of family (1) intercepts a circle on $K$ with center $z_{1}$ and radius $r_{1}$, then $r^{2}-|z|^{2}=r_{1}^{2}-\left|z_{1}\right|^{2}$. Whence $z_{1}, r_{1}$ satisfy (1), where $a$ and $\Gamma$ are replaced by their orthogonal projections onto $K$ and the parameter $b$ is also properly changed. Thus, the family of circles formed by intersections of spheres from family (1) with the plane $K$ can be defined by similar relations on $K$.

Let $q$ be the center of the circle $\delta, R$ be its radius. Take an arbitrary circle $\gamma$ of the family (1) intersecting $\delta$. Let $l$ be the line containing the common chord of $\delta$ and $\gamma$ (Fig. 5). Any point $x \in l$ has equal powers with respect to $\delta$ and $\gamma$, hence $|x-q|^{2}-R^{2}=\mid x-$ $\left.z\right|^{2}-r^{2}$. Expressing $r^{2}$ from (1) we obtain after simplifications $(x-a, z-q)=k$, where $k=\frac{R^{2}-|a|^{2}-|q|^{2}-b}{2}+(a, q)$. This linear equation on $x$ is nontrivial $(z-q$ and $k$ cannot vanish simultaneously), otherwise the circle $\gamma$ coincides with $\delta$, which contradicts the assumption. So, we obtain a family of lines $\mathcal{L}=\{l(z), z \in \Gamma\}$, where $l(z)=l=$ $\left\{x \in \mathbb{R}^{2} \mid(x-a, z-q)=k\right\}$. If $\Gamma$ is a subset of a straight line, then all the lines of $\mathcal{L}$ 


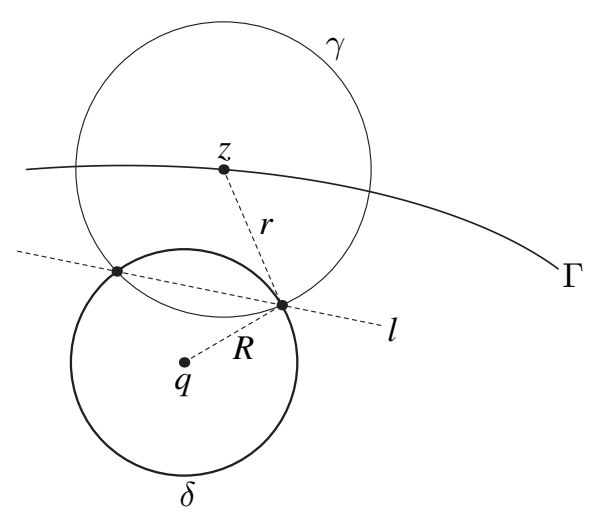

Fig. 5

concur, or they are all parallel. In this case the statement is trivial: for any point $D_{1} \in \delta$ the process has period 2, whenever it can start. If $\Gamma$ is a quadric, then all lines of the family $\mathcal{L}$ touch the quadric $\alpha$, which is obtained from the dual quadric $(\Gamma-q)^{*}$ by applying the multiplication by the factor $k$ and the translation by the vector $a$. Therefore, in this case the theorem follows from Theorem A.

In the next three sections we observe some crucial corollaries of Theorem 1. We are going to see that Theorem 1 is a quite powerful tool to prove many Poncelet type results. First, we establish a special closing theorem for spheres in the space $\mathbb{R}^{3}$ that imply, just as simple special cases, all four classical Theorems A-D. Another simple corollary of Theorem 1 is that the zigzag theorem holds for any pair of circles in $\mathbb{R}^{d}$, not necessarily in $\mathbb{R}^{3}$. Then we go further and derive a general closing theorem in $\mathbb{R}^{d}$ which is a generalization of Emch's theorem (Theorem D) for all dimensions $d \geq 2$.

\section{Closing theorem in $\mathbb{R}^{3}$ and the four classical theorems}

In the space $\mathbb{R}^{3}$ given a sphere $Q$ and spheres $S_{0}, S_{1} \subset \mathbb{R}^{3}$ that are not symmetric with respect to $Q$ (i.e. are not mapped to each other by the inversion with respect to $Q$ ). The sphere $Q$, and one of the spheres $S_{0}, S_{1}$ may become points. Let $\mathcal{M}_{i}, i=0,1$, be the corresponding families of spheres tangent to $S_{0}$ and $S_{1}$ (see the definition in Section 2). Choose $i \in\{0,1\}$ and consider the family $\mathcal{M}$ of spheres from $\mathcal{M}_{i}$ that are orthogonal to $Q$. There are at most two singular points in $\mathbb{R}^{3}$ for the family $\mathcal{M}$, this will be shown in Remark 1. Orthogonality, as usual, means that two tangent planes to the spheres drawn at their common point are perpendicular to each other. Equivalently, two radii of the spheres starting at their common point form a right angle. If $Q$ is a plane, then a sphere is orthogonal to $Q$ iff it is centered on $Q$; if $Q$ is a point, then a sphere is orthogonal to $Q$ iff it passes through $Q$.

Theorem 2. The family $\mathcal{M}$ possesses the closing property on any circle $\delta \subset \mathbb{R}^{3}$ that does not pass through singular points and does not lie on a sphere from $\mathcal{M}$. 
The geometrical meaning of this theorem is less obvious than for Theorems A-D because of that orthogonality condition. However, as we will see below, Theorem 2 implies all of them. In some sense, Theorem 2 is a common root for all the classical closing theorems.

The proof of Theorem 2 is by merely showing that the family $\mathcal{M}$ satisfies the assumptions of Theorem 1. We use several well-known facts of elementary geometry. Any pair of spheres $S_{0}, S_{1}$ of different radii has two homothety centers $h_{0}, h_{1}$. This means that there is a homothety centered at $h_{0}$ taking the sphere $S_{0}$ to $S_{1}$, and the same holds for the point $h_{1}$. For each $i=0,1$ the line joining the points of tangency of any sphere from $\mathcal{M}_{i}$ with $S_{0}$ and with $S_{1}$ passes through $h_{i}$. Moreover, the point $h_{i}$ has the same power with respect to all spheres of the family $\mathcal{M}_{i}$. In the sequel we denote this power by $p_{i}$.

Proof of Theorem 2. With possible inversion it may be assumed that $Q, S_{0}$, and $S_{1}$ are spheres (not planes) and that $S_{0}, S_{1}$ have different radii. Thus, $r_{0} \neq r_{1}$, where $S_{k}=$ $S\left(z_{k}, r_{k}\right), k=0,1$. Choose some $i \in\{0,1\}$ take the corresponding subset $\mathcal{M}$ of the family $\mathcal{M}_{i}$ and consider an arbitrary sphere $S(z, r) \in \mathcal{M}$ (Fig. 6). The power of the point

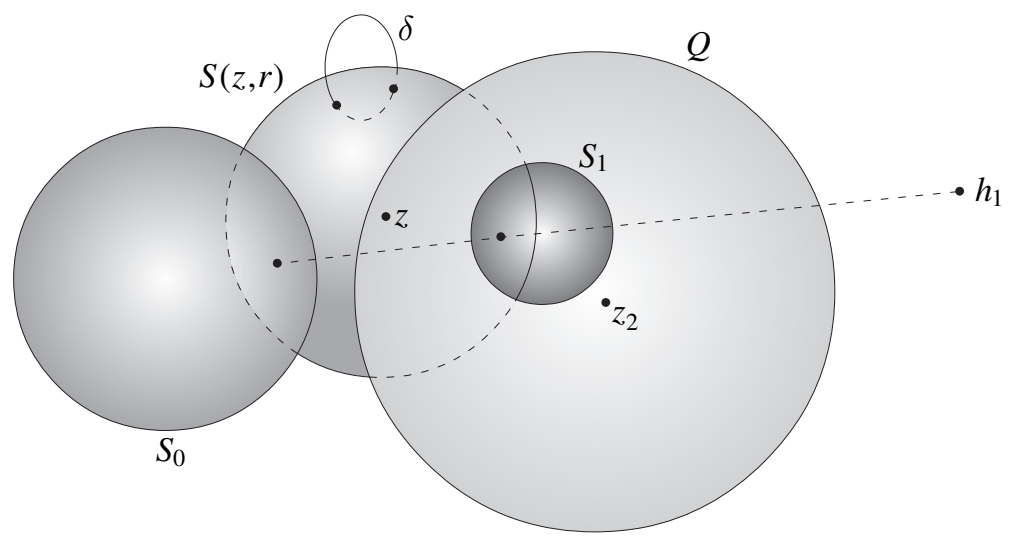

Fig. 6 Closing theorem in $\mathbb{R}^{3}$ (Theorem 2)

$h_{i}$ with respect to this sphere is equal to $p_{i}$, so $\left|z-h_{i}\right|^{2}-r^{2}=p_{i}$. Thus, the sphere $S(z, r)$ satisfies (1) with $a=h_{i}, b=-p_{i}$. Since this sphere is orthogonal to $Q$, we have $\left|z-z_{2}\right|^{2}=r^{2}+r_{2}^{2}$, where $z_{2}$ is the center of $Q$ and $r_{2}$ is its radius. Subtracting this equality from the previous one, we obtain a linear equation in $z$, which defines some plane $L \subset \mathbb{R}^{3}$. On the other hand, the centers of all spheres of $\mathcal{M}_{i}$ form a quadric in $\mathbb{R}^{3}$ with foci $z_{0}, z_{1}$. Therefore the centers $z$ of the spheres $S(z, r) \in \mathcal{M}$ lie on the intersection of that quadric with the plane $L$, i.e., on a plane quadric $\Gamma$. It now remains to apply Theorem 1.

Let us now derive Theorems A-D from Theorem 2.

Theorem $2 \Rightarrow$ Theorem A. $S_{0}, S_{1}$ are arbitrary spheres inscribed in a cone that has the quadric $\alpha$ as a plane section; $Q$ is the point at infinity.

In this case all spheres of $\mathcal{M}$ are planes, because they contain the point at infinity. They intersect the plane of the quadric $\alpha$ by lines touching $\alpha$. From this Theorem A follows. 
Theorem $2 \Rightarrow$ Theorem $\mathbf{C}$. In this case $Q$ is the plane of the circle $s$, the spheres $S_{i}$ are concentric to $s$ and have radii $|r \pm \rho|$, where $r$ is the radius of $s$.

Theorem $2 \Rightarrow$ Theorem D. We set $Q$ to be the plane of the circles $\alpha_{0}, \alpha_{1}, \delta$, the sphere $S_{i}$ has its center on the plane $Q$ and intersects it along the circle $\alpha_{i}, i=0,1$.

In this case all spheres of $\mathcal{M}$ are centered on the plane $Q$ and intersect that plane by circles tangent to both $\alpha_{0}$ and $\alpha_{1}$.

Theorem $2 \Rightarrow$ Theorem B. Theorem B is a special case of Theorem D (Section 7).

Thus, the Poncelet theorem corresponds to the case of Theorem 2, when $Q$ is the point at infinity; the zigzag theorem corresponds to the case, when $S_{0}, S_{1}$ are disjoint and both orthogonal to $Q$; finally the Emch theorem corresponds to another special case, when the circle $\delta$ lies on the sphere $Q$, and the spheres $S_{0}$ and $S_{1}$ are both orthogonal to $Q$.

Remark 1. Theorem 2 holds for general spheres $Q, S_{0}, S_{1}$ and a circle $\delta$ in the sense that any three spheres and a circle in general position satisfy the assumptions of Theorem 2 . To see this we show that there are at most two singular points for the family $\mathcal{M}$. With possible inversion we may assume that the radii of the spheres $S_{0}, S_{1}$ are different. If $z$ is a singular point, then $z \notin Q$ and, moreover, the line joining $z$ and $\tilde{z}$ (the inverse image of $z$ with respect to the sphere $Q)$ passes through $h$, and $\left(h_{i}-z, h_{i}-\tilde{z}\right)=p_{i}$. To show this we make an inversion with center at the point $z$ (the images will be denoted by prime). Spheres of $\mathcal{M}$ containing $z$ become planes passing through the center of the sphere $Q^{\prime}$ (or perpendicular to the plane $Q^{\prime}$ in case $z \in Q$ ), tangent to the spheres $S_{0}^{\prime}, S_{1}^{\prime}$. There are at most two such planes unless the center of $Q^{\prime}$ coincides with the homothety center of the spheres $S_{0}^{\prime}, S_{1}^{\prime}$. This case corresponds to the property of the point $z$ described above. There are at most two points $z$ with this property.

\section{Zigzag theorem for two circles in $\mathbb{R}^{d}$}

Another immediate corollary of Theorem 1 is the extension of the zigzag theorem to spaces of all dimensions.

Corollary 1. Theorem C holds for any pair of circles in $\mathbb{R}^{d}$ satisfying condition (c).

Proof. Let $s$ and $\delta$ be arbitrary circles in $\mathbb{R}^{d}$ and $\rho>0$ be the length of the jump. The family $\mathcal{M}$ of spheres of radius $\rho$ centered on $s$ can be defined by relations (1), where $\Gamma=s, a$ is the center of the circle $s, r$ is its radius, and $b=\rho^{2}-r^{2}$.

\section{Closing theorem for spheres in $\mathbb{R}^{d}$}

As we already mentioned in the introduction, the Emch theorem plays a special role among the four classical closing theorems. In some sense, it is the strongest one among them, because the Poncelet theorem for two circles, the Steiner theorem, and the zigzag theorem in case of circles on one plane follow easily from Emch's theorem. This will be shown in Section 7. That is why it would be most interesting to have the Emch theorem not only in the plane, but in the space $\mathbb{R}^{d}$ for any $d \geq 2$. Instead of the family of circles $\mathcal{M}$ 


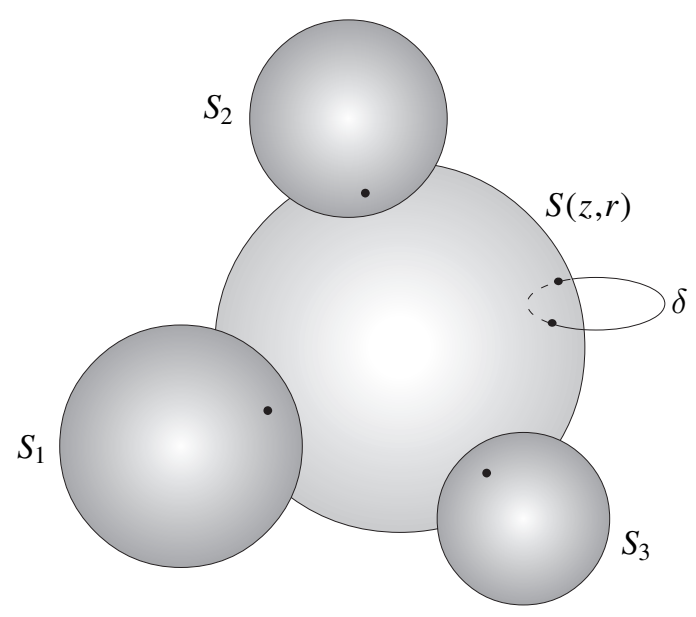

Fig. 7 Generalized Emch theorem (Theorem 3)

touching two given circles $\alpha_{0}, \alpha_{1}$ on the plane, as in Theorem D, we now consider a family of spheres touching $d$ given spheres in $\mathbb{R}^{d}$ (Fig. 7). It appears that, under some general assumptions, this family possesses the closing property on any circle $\delta \subset \mathbb{R}^{d}$. This means that if there is a closed chain of $n$ spheres in $\mathbb{R}^{d}$ touching $d$ given spheres such that each pair on neighboring spheres intersect on the circle $\delta$, then there are infinitely many such chains. Moreover, for any point of $\delta$ there is a chain starting in it. This is a generalization of Emch's theorem to $\mathbb{R}^{d}$. To formulate it one needs to overcome one difficult point. In Theorem $\mathrm{D}$ we deal with two families of circles $\mathcal{M}_{0}, \mathcal{M}_{1}$ touching two given circles. For $d$ spheres in the space $\mathbb{R}^{d}$ there may be as many as $2^{d-1}$ such families of spheres. To classify them it will be convenient to use the notion of oriented sphere.

An oriented sphere $S(z, r)$ of radius $r \in \mathbb{R}$ centered at $z$ is the set of points $x \in \mathbb{R}^{d}$ such that $|x-z|=|r|$. So the radius of an oriented sphere may take any real value; $S(z, r)$ and $S(z,-r)$ are considered as two different spheres, whenever $r \neq 0$, although they correspond to the same set of points in $\mathbb{R}^{d} . P(n, c)$ denotes the oriented plane that consists of points $x \in \mathbb{R}^{d}$ such that $(n, x)=c$. The planes $P(n, c)$ and $P(-n,-c)$ are also considered to be different, although they correspond to the same set of points. In this section all spheres and planes are assumed to be oriented. A sphere $S(z, r)$ touches a sphere $S\left(z_{0}, r_{0}\right)$ when $\left|z-z_{0}\right|=\left|r+r_{0}\right|$; it touches a plane $P(n, c)$ when $(z, n)+r=c$. A collection of spheres $S_{i}=S\left(z_{i}, r_{i}\right), i=1, \ldots, d$ is said to be in the general position if the affine hull of the points $\left(z_{i}, r_{i}\right)^{T} \in \mathbb{R}^{d+1}, i=1, \ldots, d$, is of dimension $d-1$. This means that the points $\left(z_{i}, r_{i}\right)^{T}$ are vertices of a $(d-1)$-dimensional simplex. The general position property is invariant with respect to translations and orthogonal transforms of $\mathbb{R}^{d}$, but not with respect to inversions. We call two collections of spheres (including planes) equivalent if one of them can be obtained from the other by finitely many isometries and inversions. Now we are ready to formulate the main theorem.

Let us have a collection of $d$ spheres $S_{i}=S\left(z_{i}, r_{i}\right), i=1, \ldots, d$. Some of them, but not all simultaneously, may become points. Consider the family $\mathcal{M}$ of spheres tangent to all 
the spheres $S_{i}$. Note that in case $d \geq 3$ the family $\mathcal{M}$ may be empty for some collections. For a set of $d$ usual (non-oriented) spheres there are up to $2^{d-1}$ such families, depending on the orientation.

Theorem 3. Let a collection of $d$ spheres in $\mathbb{R}^{d}$ be equivalent to a collection in general position and the family $\mathcal{M}$ of spheres touching them be nonempty. If a circle $\delta \subset \mathbb{R}^{d}$ does not contain singular points and does not lie on a sphere from $\mathcal{M}$, then $\mathcal{M}$ possesses the closing property on $\delta$.

Let us see what Theorem 3 gives for small dimensions $d$.

$\boldsymbol{d}=\mathbf{2}$. Any pair of distinct circles in the plane is in general position, therefore Theorem 3 becomes the Emch theorem.

$\boldsymbol{d}=3$. Theorem 3 holds for any triple of spheres in $\mathbb{R}^{3}$, for which there is a sphere touching them. If a triple $S\left(z_{i}, r_{i}\right), i=1,2,3$, is not in general position, then the points $\left(z_{i}, r_{i}\right)^{T} \in \mathbb{R}^{4}$ are collinear. Whence the centers $z_{i}$ are on the same line as well. Applying the inversion with some center outside that line we get spheres in general position.

Thus, in case of small dimensions any collection of spheres are equivalent to one in general position. For $d \geq 4$ this may not be the case. Nevertheless, a "typical" set of $d$ spheres and one circle in $\mathbb{R}^{d}$ does satisfy the assumptions of Theorem 3 . Let us first establish this, and then we prove the theorem. We start with three auxiliary results. Observe that any sphere $S(z, r) \in \mathcal{M}$ satisfies the following system of equations:

$$
r^{2}=|z|^{2}-2\left(z_{i}, z\right)-2 r_{i} r+\left|z_{i}\right|^{2}-r_{i}^{2}, \quad i=1, \ldots, d ;
$$

and any plane $P(n, c) \in \mathcal{M}$ satisfies the system

$$
\left(z_{i}, n\right)+r_{i}=c, \quad i=1, \ldots, d .
$$

Lemma 1. For any collection of spheres in general position the family $\mathcal{M}$ contains at most two planes.

Proof. Subtracting the first equation of system (3) from the others we obtain the linear system $\left(z_{i}-z_{1}, n\right)=r_{i}-r_{1}, i=2, \ldots, d$ of rank $d-1$. Its solutions $n$ form a straight line in $\mathbb{R}^{d}$, which contains at most two points such that $|n|=1$.

Planes of the family $\mathcal{M}$, if they exist at all, are limits for the spheres of that family. Now we clarify when all spheres forming an affine plane (in the space of spheres) can touch one sphere.

Lemma 2. Suppose an affine plane $L \subset \mathbb{R}^{d+1}$, $\operatorname{dim} L \geq 1$ is such that there is a sphere (or a point) $S_{0} \subset \mathbb{R}^{d}$ tangent to every sphere of the family $\mathcal{L}=\left\{S(z, r) \mid(z, r)^{T} \in L\right\}$. Then $L$ is a line and $\mathcal{L}$ is a pencil of spheres tangent at one point. Moreover, $S_{0} \in \mathcal{L}$.

Proof. If $\operatorname{dim} L=1$, then the center $z$ of any sphere of $\mathcal{L}$ lies on a fixed straight line $b \subset \mathbb{R}^{d}$ and its radius $r$ is a linear function of $z$. If all the spheres from $\mathcal{L}$ touch $S_{0}$, then the center of $S_{0}$ lies on $b$ as well, otherwise $r$ is not linear in $z$. Therefore, $\mathcal{L}$ is a pencil of tangent spheres. In particular, $S_{0}$ also belongs to $\mathcal{L}$. In case $\operatorname{dim} L \geq 2$ all lines on $L$ concur at one point corresponding to the sphere $S_{0}$, which is impossible. 
The last auxiliary result restricts the location of singular points of the family $\mathcal{M}$.

Lemma 3. For a collection of $d$ spheres in general position all singular points of the family $\mathcal{M}$ lie on some affine plane $E$ of dimension $d-2$. If $\mathcal{M}$ contains two planes, then their intersection coincides with $E$.

Proof. It suffices to show that if a point $z_{0}$ is singular, then the point $\left(z_{0}, 0\right)^{T}$ belongs to a plane $E_{0} \subset \mathbb{R}^{d+1}$, which is an affine hull of the points $\left(z_{i}, r_{i}\right)^{T}, i=1, \ldots, d$. Note that $\operatorname{dim} E_{0}=d-1$. For a sphere $S(z, r)$ that passes through the point $z_{0}$ we have $r^{2}=|z|^{2}-2\left(z_{0}, z\right)+\left|z_{0}\right|^{2}$. Subtracting this equation from each equation of (2) we obtain the system

$$
\left(z_{i}-z_{0}, z\right)+r_{i} r=\frac{1}{2}\left(\left|z_{i}\right|^{2}-r_{i}^{2}-\left|z_{0}\right|^{2}\right), \quad i=1, \ldots, d
$$

If $\left(z_{0}, 0\right)^{T} \notin E_{0}$, then the matrix of this system has full rank $d$. Therefore its solutions, i.e., points $(z, r)^{T}$, form a straight line $l \in \mathbb{R}^{d+1}$. Substituting the solutions in system (2) we get a quadratic equation. If all its coefficients vanish, then all the spheres corresponding to the solutions $(z, r)^{T}$ touch the sphere $S_{1}$. Hence by Lemma 2 they constitute a pencil of tangent spheres. Then all the spheres $S_{i}$ belong to this pencil, therefore all the points $\left(z_{i}, r_{i}\right)^{T}$ lie on a line, which contradicts to their general position. Thus, the obtained quadratic equation is nontrivial and has at most two solutions. So, there are at most two spheres from $\mathcal{M}$ passing through $z_{0}$. Whence, if a point $z_{0}$ does not belong to a plane from $\mathcal{M}$, then it is nonsingular. If it belongs to a plane from $\mathcal{M}$, then by (3) it satisfies the equations $\left(z_{i}-z_{0}, n\right)-r_{i}=0, i=1, \ldots, d$. This system has full rank $d$ and so it has at most one solution $n$. Comparing this system with (4) we conclude that the line $l$ is parallel to the vector $(n, 1)^{T} \in \mathbb{R}^{d+1}$. Substituting the solutions $(z, r) \in l$ in the first equation of (2) and taking into account that $|n|=1$ we get a linear equation (quadratic terms disappear), which has at most one solution. Thus, there is a unique plane and at most one sphere from $\mathcal{M}$ passing through $z_{0}$.

Thus, if a circle $\delta$ does not intersect the $(d-2)$-dimensional plane $E$, then it does not contain singular points. For an arbitrary point $z \in \delta$ there are at most two spheres from $\mathcal{M}$ passing through $z$. If the circle $\delta$ is not contained in any of these two spheres, then it does not lie on any sphere of $\mathcal{M}$. Thus, we see that in general position a set of $d$ spheres and one circle in $\mathbb{R}^{d}$ indeed satisfy the assumptions of Theorem 3 . Now we can prove the theorem.

Proof of Theorem 3. It suffices to consider the case of $d$ spheres in general position. We show that the family $\mathcal{M}$ can be defined by relations (1) and then apply Theorem 1 . The proof will be realized for spheres of $\mathcal{M}$, for planes (if they exist) it will follow by the limit passage. Assume first that among the spheres $S_{i}$ there are two ones of different radii (regarding the sign). Subtracting the first equation of system (2) from the others we obtain $d-1$ linear equations $2\left(z_{1}-z_{i}, z\right)+2\left(r_{1}-r_{i}\right) r=\alpha_{i}, i=2, \ldots, d$, where $\alpha_{i}$ are some constants. For at least one of them the coefficient $r_{1}-r_{i}$ does not vanish. Expressing $r=r(z)$ from that equation and substituting it in the other equations, we get a system 
of $d-2$ linear equations (of rank $d-2$ ) for the variable $z$. Its solutions $z$ form a twodimensional affine plane $L_{0} \subset \mathbb{R}^{d}$. Since the function $r(z)$ is linear, the points $(z, r(z))^{T}$ form a two-dimensional affine plane $L \subset \mathbb{R}^{d+1}$. Substituting $r(z)$ in the first equation of system (2) we obtain an equation for $z$, which is either quadratic or linear. If all points of $L_{0}$ satisfy it, then all the spheres associated to the points $(z, r)^{T} \in L$ touch the sphere $S_{1}$, which is impossible (Lemma 2). Therefore points $z \in L_{0}$ satisfying that equation form a plain quadric (or a line) $\Gamma \subset L_{0}$. Now substitute $r(z)$ in the right hand side of the first equation (2) and obtain $r^{2}=|z|^{2}-(a, z)+c$, where $a \in \mathbb{R}^{d}$ and $c \in \mathbb{R}$. Extracting the perfect square we arrive at (1). Finally, if the radii $r_{i}$ are all the same, then the centers of the spheres of $\mathcal{M}$ lie on a straight line $\Gamma$, and one can easily write equation (1).

\section{Elementary proof of Theorem D}

Now we come back to the planar Emch theorem (Theorem D). Among the four classical closing theorems it is the strongest one: the three others are actually its special cases. Indeed, if the circle $\alpha_{1}$ degenerates into a point, then an inversion with the center at this point yields Theorem A for two circles. If $\alpha_{0}$ lies within $\alpha_{1}$, and the circle $\delta$ is orthogonal to all circles of the family $\mathcal{M}_{1}$, then we obtain Theorem B. We use the fact that the center $h_{1}$ of homothety of the circles $\alpha_{0}$ and $\alpha_{1}$ has the same power $p_{1}$ with respect to all circles of $\mathcal{M}_{1}$ (see Section 3). Therefore the circle $\delta$ of radius $\sqrt{p_{1}}$ centered at $h_{1}$ is orthogonal to all circles of $\mathcal{M}_{1}$ and contains all their points of tangency. Hence, Theorem $\mathrm{B}$ follows from Theorem D. Finally, if the circles $\alpha_{0}$ and $\alpha_{1}$ are concentric, then we arrive at Theorem $\mathrm{C}$ for the case when the circles $\delta$ and $s$ are on one plane. Indeed, if we take as $\alpha_{0}, \alpha_{1}$ the circles of radii $|r \pm \rho|$ concentric to $s$ ( $r$ is the radius of $s$ ), then we obtain Theorem $\mathrm{C}$.

Thus, for concentric circles $\alpha_{0}, \alpha_{1}$ Theorem D becomes the "planar" version of the zigzag theorem; in case when $\alpha_{0}$ is the point at infinity, we obtain the Poncelet theorem for two circles; finally, if $\alpha_{0}$ is inside $\alpha_{1}$, and $\delta$ is orthogonal to all circles of the family $\mathcal{M}_{1}$, then we get the Steiner theorem.

The question arises if it is possible to give a proof of Theorem $\mathrm{D}$ that will be elementary and autonomous (not relying on the Poncelet theorem, in contrast to the proofs of Theorems 2 and 3). In this section we give such a proof using only tools of elementary geometry, the most complicated of which are inversions and pencils of circles. To avoid technical difficulties we restrict ourselves to the following case of mutual position of circles in Theorem D:

(d) The circle $\delta$ is inside $\alpha_{1}$, the circle $\alpha_{0}$ is inside $\delta$, and we consider the family $\mathcal{M}_{1}$ of circles touching $\alpha_{1}$ from inside and $\alpha_{0}$ from outside.

The idea of the proof is the following: let a chain of circles inscribed in the annulus bounded by $\alpha_{0}$ and $\alpha_{1}$ intersect the circle $\delta$ in successive points $D_{1}, D_{2}, \ldots$ and another chain of circles inscribed in the annulus intersect $\delta$ in points $D_{1}^{\prime}, D_{2}^{\prime}, \ldots$ For any $i=1,2, \ldots$ draw a new circle $s_{i}$ tangent to $\alpha_{0}$ through the points $D_{i}$ and $D_{i}^{\prime}$. It appears that all the new circles $s_{i}, i=1,2, \ldots$, touch some circle $c$ (Fig. 8).

Moreover, $c, \delta$ and $\alpha_{1}$ belong to one pencil of circles. This implies that if the first chain cycles $\left(D_{n+1}=D_{1}\right)$, then the second also does $\left(D_{n+1}^{\prime}=D_{1}^{\prime}\right)$. The idea is quite clear, but 


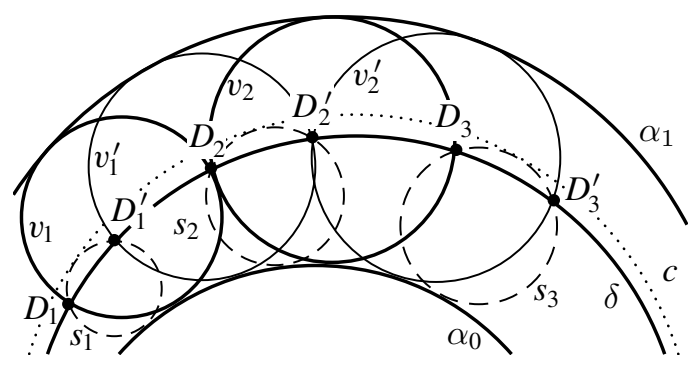

Fig. 8

to realize it we need to establish several auxiliary results. All of them are elementary, but quite technical. Some of these results, such as Theorem 4 and Proposition 1 are, probably, of some independent interest.

We begin with a simple auxiliary fact. In the sequel we assume that an $\operatorname{arc} B$ of a circle has the positive direction from $A$ to $B, A B$ denotes the angle defined by that arc.

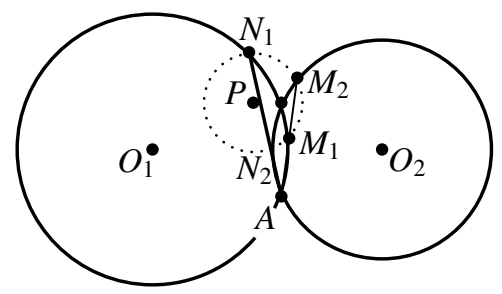

Fig. 9

Lemma 4. Two circles of radii $r_{1}, r_{2}$ centered at $O_{1}, O_{2}$ intersect at points $A$ and $B$. Suppose $P$ is the fourth vertex of the parallelogram $O_{2} A O_{1} P$; then for any circle centered at $P$ intersecting the first circle at some points $M_{1}, N_{1}$, and the second one at points $M_{2}$, $\mathrm{N}_{2}$ (Fig. 9) the following hold:

a) The lines $M_{1} M_{2}$ and $N_{1} N_{2}$ pass through $A$.

b) $\frac{M_{1} N_{1}}{M_{2} N_{2}}=\frac{A N_{1}}{A M_{2}}=\frac{A M_{1}}{A N_{2}}=\frac{B M_{1}}{B M_{2}}=\frac{B N_{1}}{B N_{2}}=\frac{r_{1}}{r_{2}}$.

Proof. a) The triangles $P O_{1} M_{1}$ and $M_{2} O_{2} P$ are equal by three equal sides, hence $\angle M_{1} O_{1} P=\angle M_{2} O_{2} P$. Furthermore, $\angle P O_{1} B=\angle P O_{2} B$, since $O_{2} O_{1} P B$ is an equilateral trapezium. Subtracting the second equality from the first one, we obtain $\angle M_{1} O_{1} B=$ $\angle M_{2} O_{2} B$, therefore $B \breve{M}_{1}=B \breve{M}_{2}$. Consequently, $\angle M_{1} A B=\angle M_{2} A B$, and thus the line $M_{1} M_{2}$ passes through $A$. The proof for $N_{1} N_{2}$ is the same.

b) It follows from a) that the chords $M_{1} N_{1}$ and $M_{2} N_{2}$ define equal angles on the two circles: $\angle M_{1} A N_{1}=\angle M_{2} A N_{2}$. Whence $\frac{M_{1} N_{1}}{M_{2} N_{2}}=\frac{r_{1}}{r_{2}}$. Similarly, $\frac{B M_{1}}{B M_{2}}=\frac{B N_{1}}{B N_{2}}=\frac{r_{1}}{r_{2}}$. Finally, since the quadrangle $M_{1} M_{2} N_{1} N_{2}$ is inscribed in a circle, it follows that the 
triangles $M_{1} A N_{1}$ and $N_{2} A M_{2}$ are similar with the factor $\frac{M_{1} N_{1}}{M_{2} N_{2}}=\frac{r_{1}}{r_{2}}$. Therefore $\frac{A N_{1}}{A M_{2}}=\frac{A M_{1}}{A N_{2}}=\frac{r_{1}}{r_{2}}$.

Before formulating the crucial auxiliary fact, Theorem 4, let us recall that a pencil of circles is a set of circles on the plane orthogonal to two different circles (all circles may degenerate into lines and points, unless the opposite is stated). Circular pencils are straight lines in the three-dimensional space of circles. Any pair of circles $\mathbf{b}, \mathbf{c} \subset \mathbb{R}^{2}$ is contained in a unique pencil that will be denoted by $\mathcal{P}\{\mathbf{b}, \mathbf{c}\}$. For every $t \in \mathbb{R} \cup\{\infty\}$ the set of points on the plane, for which the ratio of powers with respect to given circles $\mathbf{b}$ and $\mathbf{c}$ equals to $t$ is either empty or a circle of the pencil $\mathcal{P}\{\mathbf{b}, \mathbf{c}\}$.

Now we are going to establish the main theorem. Take two circles with a common center $P$, we call them the bigger circle and the smaller one. Consider the families of circles $\mathcal{M}_{j}$, $j=0,1$, touching them. Take an arbitrary pair $\beta_{0}, \beta_{1} \in \mathcal{M}_{0}$ and a pair $\gamma_{0}, \gamma_{1} \in \mathcal{M}_{1}$. The points of intersection of $\beta_{i}$ and $\gamma_{k}$ will be denoted by $A_{i k}^{0}$ and $A_{i k}^{1}$ (the first point is farther from the center $P$ than the second one). Finally, draw one more circle centered at $P$. Let it meet each of the circles $\beta_{i}$ and $\gamma_{k}$ at two points $b_{i}^{s}, s=0,1$ (respectively $c_{k}^{s}$ ). If a point goes around the circle $\beta_{i}$ counterclockwise from its point of tangency with the bigger circle, then it meets the point $b_{i}^{0}$ first and $b_{i}^{1}$ second. The same with the points $c_{k}^{s}$ (Fig. 10). In the notation of Theorem 4 all superscripts are taken modulo 2, for example, $A_{10}^{2}=A_{10}^{0}, c_{1}^{3}=c_{1}^{1}$.

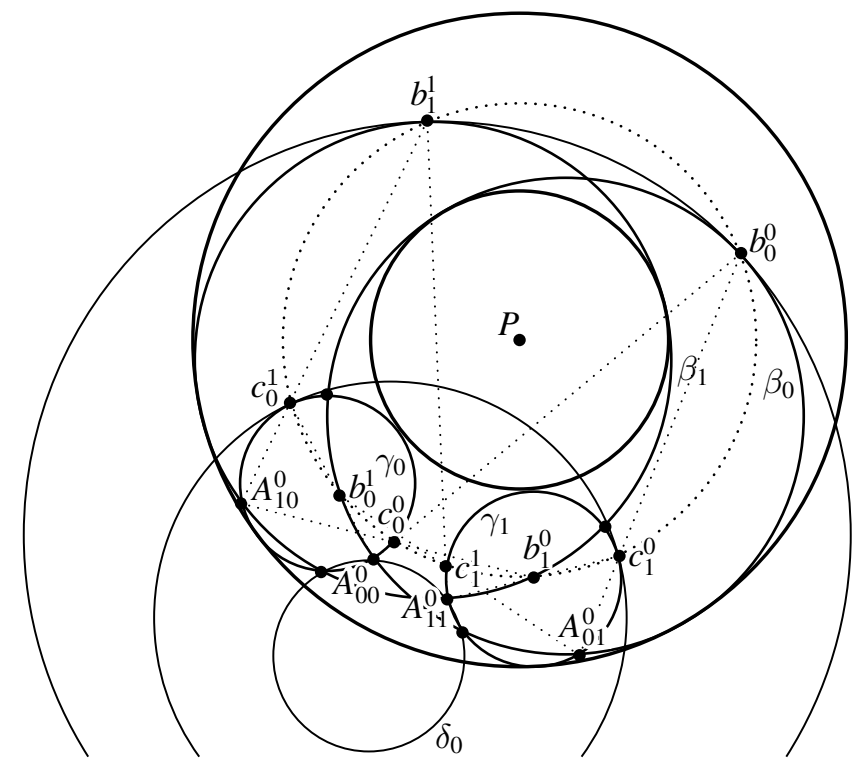

Fig. 10

Theorem 4. Given two circles with a common center $P$ and arbitrary circles $\beta_{i} \in \mathcal{M}_{0}$, $\gamma_{k} \in \mathcal{M}_{1}, i, k=0,1$. Suppose $A_{i k}^{s}$ are the corresponding 8 points of their intersection; then 
a) The 4 points $A_{i k}^{i+k}, i, k \in\{0,1\}$, lie on one circle (denote it by $\delta_{0}$ ). The same holds for the 4 points $A_{i k}^{i+k+1}, i, k \in\{0,1\}$ (a circle $\delta_{1}$ ).

b) Let an arbitrary circle centered at $P$ meet $\beta_{i}, \gamma_{k}$ at points $b_{i}^{s}, c_{k}^{s}$, respectively. Then for every $i, k \in\{0,1\}$ the lines $b_{i}^{0} c_{k}^{0}$ and $b_{i}^{1} c_{k}^{1}$ pass through the point $A_{i k}^{0}$.

c) Choose arbitrary $q, j \in\{0,1\}$ and draw a circle touching the circles $\beta_{i}$ at the points $b_{i}^{i+q}, i=0,1$, and a circle touching the circles $\gamma_{k}$ at the points $c_{k}^{k+q+j+1}, k=0,1$. Then these two circles belong to one pencil with $\delta_{j}$.

Thus, if four circles $\beta_{i}, \gamma_{k}, i, k \in\{0,1\}$, touch two concentric circles, then the eight points of their intersection $A_{i k}^{s}$ are naturally split into two quadruples, each of them lies on one circle. Drawing a third concentric circle we get the 8 additional points of intersection $b_{i}^{s}$, $c_{k}^{s}$ that form 8 triples of collinear points (Fig. 10). Finally, if we consider two pairs of circles touching $\beta_{i}, \gamma_{k}$ at the points of their intersection with the third circle, then we obtain 4 triples of circles, each of them is in one pencil. So this configuration of seven circles produces two circles, 8 lines and 4 pencils.

Proof of Theorem 4. Let $R$ and $r$ be radii of the circles $\beta_{0}$ and $\gamma_{0}$, respectively, and $O_{1}$, $\mathrm{O}_{2}$ be their centers. Then $O_{2} A_{00}^{0} O_{1} P$ is a parallelogram with sides of lengths $r$ and $R$. By Lemma 4 the lines $b_{0}^{0} c_{0}^{0}$ and $b_{0}^{1} c_{0}^{1}$ concur at $A_{00}^{0}$. This proves item $\mathrm{b}$ ) for $i=k=0$, the proof for the other indices is the same.

Now draw a circle $\mathbf{b}$ touching $\beta_{0}$ and $\beta_{1}$ at the points $b_{0}^{0}$ and $b_{1}^{1}$, respectively, and a circle $\mathbf{c}$ touching $\gamma_{0}$ and $\gamma_{1}$ at the points $c_{0}^{1}$ and $c_{1}^{0}$. We assume that both $\mathbf{b}$ and $\mathbf{c}$ do not degenerate into lines. Denote by $x$ the radius of the circle $\mathbf{b}$ taken with the sign: it is positive if this circle touches $\beta_{0}$ from outside, and negative otherwise. Similarly $y$ is the radius of $\mathbf{c}$ with the sign. Denote also by $B$ the second point of intersection of the line $b_{0}^{0} A_{00}^{0}$ with the circle $\mathbf{b}$, and by $C$ the second point of intersection of the line $c_{0}^{1} A_{00}^{0}$ with the circle $\mathbf{c}$. The similarity of circles implies that $b_{0}^{0} B=\frac{x}{R} b_{0}^{0} A_{00}^{0}$. Therefore the power of the point $A_{00}^{0}$ with respect to the circle $\mathbf{b}$ is $A_{00}^{0} b_{0}^{0} \cdot A_{00}^{0} B=\left(1+\frac{x}{R}\right)\left(A_{00}^{0} b_{0}^{0}\right)^{2}$. Similarly, the power of the point $A_{00}^{0}$ with respect to $\mathbf{c}$ is $\left(1+\frac{y}{r}\right)\left(A_{00}^{0} c_{0}^{1}\right)^{2}$. Lemma 4 yields $A_{00}^{0} b_{0}^{0} / A_{00}^{0} c_{0}^{1}=R / r$. Therefore, the ratio of the powers of the point $A_{00}^{0}$ with respect to the circles $\mathbf{b}$ and $\mathbf{c}$ is equal to $\frac{(R+x) R}{(r+y) r}$. In the same way we obtain that for each of the points $A_{11}^{0}, A_{10}^{1}, A_{01}^{1}$ the ratio of powers with respect to $\mathbf{b}$ and $\mathbf{c}$ also equals $\frac{(R+x) R}{(r+y) r}$. Whence, these 4 points are on one circle $\left(\delta_{0}\right)$ that belongs to the pencil $\mathcal{P}\{\mathbf{b}, \mathbf{c}\}$. This proves items a) and c) for $j, q=0$. The proof for other $j, q$ is the same.

Corollary 2. Under the assumptions of Theorem 4 for any $j=0,1$ the following holds: For an arbitrary circle $\mathbf{b}$ touching the circles $\beta_{0}$ and $\beta_{1}$ in the same way (both from inside or both from outside) there exists a circle $\mathbf{c} \in \mathcal{P}\left\{\delta_{j}, \mathbf{b}\right\}$ touching $\gamma_{0}$ and $\gamma_{1}$ in the same way.

Remark 2. Through any point $A$ of the circle $\gamma_{0}$ (different from points of its tangency with the bigger circle and with the smaller one) one can draw two circles of the family $\mathcal{M}_{0}$. Precisely for one of them $A$ is the closest to $P$ point of intersection with $\gamma_{0}$. 
Theorem $4 \mathrm{~b}$ ) implies, in particular, that for any $i, k \in\{0,1\}$ the line joining the points of tangency of $\beta_{i}, \gamma_{k}$ with the bigger circle passes through the point $A_{i k}^{0}$. The same is true for the smaller circle. We need the converse, whose proof will be a simple exercise for the reader.

Lemma 5. Suppose an arbitrary straight line passing through the point of tangency of given circles $\alpha$ and $\beta$ meet these circles at points $A$ and $B$, respectively. The circle $s$ passes through $B$ and touches $\alpha$ at the point A. Then all such circles s touch a fixed circle different from $\alpha$. This circle touches $\beta$ and is concentric to $\alpha$.

Now we are formulating the assertion, from which Theorem D follows immediately. Consider arbitrary circles $\alpha_{0}, \alpha_{1}$, and $\delta$ satisfying condition (d). Take two series of circles $\left\{v_{k}\right\},\left\{v_{k}^{\prime}\right\} \subset \mathcal{M}_{1}$ and the corresponding points $\left\{D_{k}\right\}$ and $\left\{D_{k}^{\prime}\right\}$ on the circle $\delta$. We assume that these sequences both go around $\delta$ in positive direction and that $D_{1}^{\prime}$ belongs to the arc $D_{1} D_{2}$ (Fig. 8). Denote by $s_{k}$ the circle passing through the points $D_{k}, D_{k}^{\prime}$ and tangent to $\alpha_{0}$ from outside.

Proposition 1. All the circles $s_{k}, k \in \mathbb{N}$, touch a fixed circle of the pencil $\mathcal{P}\left\{\delta, \alpha_{1}\right\}$.

Proof. It consists of the consecutive application of Theorem 4 to the pairs of circles $v_{k}$, $v_{k}^{\prime}$ and $s_{k}, s_{k+1}$ for all $k \geq 1$ (Fig. 8). A suitable inversion maps the pairs $v_{1}, v_{1}^{\prime}$ and $s_{1}$, $s_{2}$ to the pairs $\beta_{0}, \beta_{1}$ and $\gamma_{0}, \gamma_{1}$ from Theorem 4 . To see this we make an inversion with center at the second point of intersection of the circles $M B K$ and $M C L$ (the first point of their intersection is $M$ ), where $K, M, L$ are the points of tangency of the circle $\alpha_{0}$ with $s_{1}, s_{2}, v_{1}$, respectively, and $B$ and $C$ are the second points of intersection of the circle $v_{1}$ with the circles $s_{1}$ and $s_{2}$, respectively. By Lemma 5 the images of the circles $s_{1}, s_{2}, v_{1}$ touch two concentric circles, one of them is the image of $\alpha_{0}$. In order to be defined we assume that the image of $\alpha_{0}$ is the smaller concentric circle and that the images of $s_{1}, s_{2}$ are situated between these concentric circles. Thus, $s_{1}, s_{2}, v_{1}$ are mapped to the circles $\gamma_{0}, \gamma_{1}, \beta_{0}$, and the points $D_{1}$ and $D_{2}$ are mapped to $A_{00}^{1}$ and $A_{01}^{0}$, respectively. Let $X_{1}$, $X_{2}$ be images of the points $D_{1}^{\prime}, D_{2}^{\prime}$. Draw a circle $\beta_{1}$ through $X_{2}$ that touches both these concentric circles from within so that $X_{2}$ is the closest (to the center) point of intersection of $\gamma_{1}$ and $\beta_{1}$ (Remark 2). Thus, $X_{2}=A_{11}^{1}$. By Theorem 4 a) the points $A_{00}^{1}, A_{01}^{0}, A_{11}^{1}$, $A_{10}^{0}$ lie on a circle. By the assumptions $A_{00}^{1}, A_{01}^{0}, A_{11}^{1}, X_{1}$ are on a circle as well. Whence $A_{10}^{0}=X_{1}$, and $\beta_{1}$ is the image of $v_{1}^{\prime}$. Thus, an inversion takes circles $v_{1}, v_{1}^{\prime}, s_{1}, s_{2}$ to the circles $\beta_{0}, \beta_{1}, \gamma_{0}, \gamma_{1}$, respectively. According to Corollary 2 the pencil $\mathcal{P}\left\{\delta, \alpha_{1}\right\}$ contains some circle $\mathbf{c}$ touching the circles $s_{1}, s_{2}$ in the same way. It is located between the circles $\delta$ and $\alpha_{1}$. Indeed, if we fix the points $D_{1}, D_{2}$ and move the point $D_{1}^{\prime}$ along the $\operatorname{arc} D_{1} D_{2}$, then at the extreme positions we have: $\mathbf{c}=\delta$ in case $D_{1}^{\prime}=D_{1}$, and $\mathbf{c}=\alpha_{1}$ in case $D_{1}^{\prime}=D_{2}$. The circle c changes continuously in the same pencil $\mathcal{P}\left\{\delta, \alpha_{1}\right\}$ when $D_{1}^{\prime}$ moves. Hence if for some $D_{1}^{\prime}$ the circle $\mathbf{c}$ is not between $\delta$ and $\alpha_{1}$, then for some interior point $D_{1}^{\prime}$ of the arc $D_{1} D_{2}$ one has either $\mathbf{c}=\delta$ or $\mathbf{c}=\alpha_{1}$. Neither of these cases is possible, because for any interior point of the arc $D_{1} D_{2}$ the circle $s_{1}$ touches neither $\delta$ nor $\alpha_{1}$.

Taking the next pairs of circles $v_{2}, v_{2}^{\prime}$ and $s_{2}, s_{3}$, we obtain a circle that belongs to the same pencil $\mathcal{P}\left\{\delta, \alpha_{1}\right\}$, lies between $\delta$ and $\alpha_{1}$, and touches both $s_{2}$ and $s_{3}$. This circle coincides 
with $\mathbf{c}$ because the pencil $\mathcal{P}\left\{\delta, \alpha_{1}\right\}$ has at most one circle between $\delta$ and $\alpha_{1}$ that touches $s_{2}$. Hence $\mathbf{c}$ touches $s_{3}$. Thus we consecutively prove that $\mathbf{c}$ is tangent to all the circles $s_{k}$.

Proof of Theorem $D$ in case (d). Let $v_{1}, \ldots, v_{n}$ be a periodic series of circles $\left(v_{n+1}=\right.$ $\left.v_{1}\right), D_{1}, \ldots, D_{n}$ be the corresponding points on $\delta$. Take an arbitrary series $v_{1}^{\prime}, v_{2}^{\prime}, \ldots$ and the corresponding sequence of points $D_{1}^{\prime}, D_{2}^{\prime}, \ldots$ With possible renumbering it may be assumed that these sequences both go around the circle $\delta$ in positive direction and that the point $D_{1}^{\prime}$ is located on the $\operatorname{arc} D_{1} D_{2}$ (Fig. 11). Consider the circles $s_{k}, k \geq 1$, from Proposition 1 and conclude that they are all tangent to a circle $\mathbf{c} \in \mathcal{P}\left\{\delta, \alpha_{1}\right\}$. The $\operatorname{arc} D_{1} D_{2}$ has only one point $D_{1}^{\prime}$, for which the circle passing through the points $D_{1}^{\prime}$ and $D_{n+1}=D_{1}$ and touching $\alpha_{0}$ from outside is tangent to c. Therefore $D_{n+1}^{\prime}=D_{1}^{\prime}$ and $s_{n+1}=s_{1}$, which completes the proof.

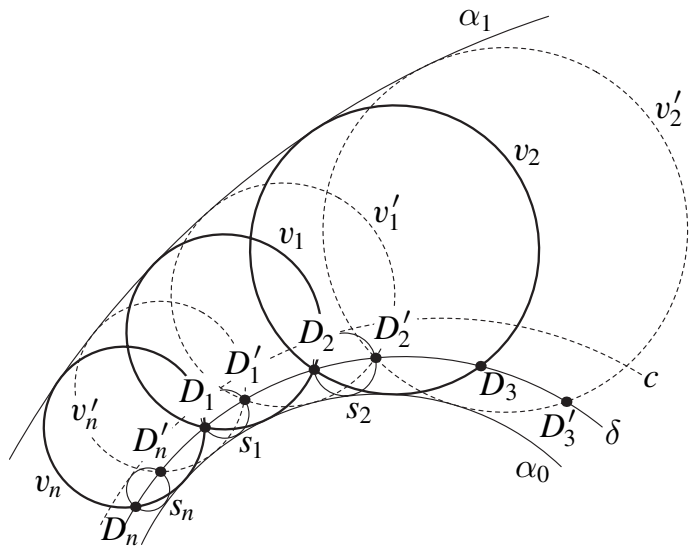

Fig. 11 Proof of Theorem D

\section{Generalization of Theorem D}

The method developed in the previous section makes it possible to go a bit further and to obtain a generalization of the Emch theorem for several pencils of circles analogous to the great Poncelet theorem [3, Theorem 16.6.7]. We formulate it only for one case of mutual position of circles. Let us have a circle $\delta$ and two sequences of circles $\left\{\alpha_{0}^{k}\right\}$ and $\left\{\alpha_{1}^{k}\right\}$. Each sequence $\left\{\alpha_{i}^{k}\right\}$ is contained in a pencil $\mathcal{A}_{i}, i=0,1$, that also contains the circle $\delta$. We assume that the circles $\left\{\alpha_{0}^{k}\right\}$ are all inside $\delta$ and that $\delta$ is inside all the circles $\left\{\alpha_{1}^{k}\right\}$. By $\mathcal{M}_{1}^{k}$ we denote the families of circles tangent to $\alpha_{1}^{k}$ and $\alpha_{0}^{k}$ with index 1 (touching $\alpha_{0}^{k}$ from without and $\alpha_{1}^{k}$ from within).

We choose an arbitrary point $D_{1} \in \delta$ and draw a circle $v_{1} \in \mathcal{M}_{1}^{1}$ through it. Then take the second point $D_{2}$ of intersection of $v_{1}$ with the circle $\delta$, draw a circle $v_{2} \in \mathcal{M}_{1}^{2}$ through it, etc. The circles $v_{k}$ are chosen in each iteration so that the sequence $\left\{D_{k}\right\}$ goes along $\delta$ in positive direction. 
Theorem 5. If the process starting at some point $D_{1}$ has period $n \geq 3$ and all the points $D_{1}, \ldots, D_{n}$ are different, then it has the same period for any initial point.

The proof is literally the same as the proof of Theorem D and is based on the following analog of Proposition 1. Given two series of circles $\left\{v_{k}\right\},\left\{v_{k}^{\prime}\right\}$, where $v_{k}, v_{k}^{\prime} \in \mathcal{M}_{1}^{k}, k \in \mathbb{N}$, and the corresponding sequences of points $\left\{D_{k}\right\},\left\{D_{k}^{\prime}\right\}$ on $\delta$. Assume that $D_{1}^{\prime}$ is located on the arc $D_{1} D_{2}$. Choose an arbitrary circle $\alpha_{0} \in \mathcal{A}_{0}$ lying inside $\delta$ and denote by $s_{k}$ the circle passing through the points $D_{k}, D_{k}^{\prime}$ and touching $\alpha_{0}$ from outside. Then all the circles $s_{k}$, $k \in \mathbb{N}$, are tangent to one circle of the pencil $\mathcal{A}_{0}$. The proof of this fact is realized in the same way as for Proposition 1 and we leave it to the reader.

\title{
Acknowledgement
}

The author is grateful to the anonymous referee for valuable comments and remarks, and to A. Akopyan and E. Gorskaya for their help in making illustrations.

\section{References}

[1] Barth, W.; Bauer, T.: Poncelet theorems. Exposition. Math. 14 (1996), 125-144.

[2] Barth, W.; Michel, J.: Modular curves and Poncelet Polygons. Math. Ann. 295 (1993), 25-49.

[3] Berger, M.: Géométrie. CEDIC, Paris 1977.

[4] Black, W.L.; Howland, H.C.; Howland, B.: A theorem about zigzags between two circles. Amer. Math. Monthly 81 (1974), 754-757.

[5] Bottema, O.: Ein Schliessungssatz für zwei Kreise. Elem. Math. 20 (1965), 1-7.

[6] Burskii, V.; Zhedanov, A.: The Dirichlet and the Poncelet problems. RIAM Symp. 2004, 22-24.

[7] Emch, A.: An application of elliptic functions to Peaucellier's link-work (inversor). Ann. of Math. (2) 2 (1901), 60-63.

[8] Griffiths, P.A.; Harris, J.: On Cayley's explicit solution to Poncelet's porism. Enseign. Math. 24 (1978), $31-40$.

[9] Hraskó, A.: Poncelet-type problems, an elementary approach. Elem. Math. 55 (2000), 45-62.

[10] Kozlov, V.V.: Rationality conditions for the ratio of elliptic integrals and the great Poncelet theorem. Moscow Univ. Math. Bull. 58 (2003) 4, 1-7.

[11] Lebesque, H.: Les Coniques. Cauthier-Villars, Paris 1942.

[12] Poncelet, J.V.: Traité des propriétés projectives des figures. Paris 1865 (first ed. in 1822).

[13] Protasov, V.: One generalization of Poncelet's theorem. Russian Math. Surveys 61 (2006) 6, 187-188.

[14] Schoenberg, I.J.: On Jacobi-Bertrand's Proof of a Theorem of Poncelet. Studies in Pure Mathematics, Birkhäuser, Boston 1983, 623-627.

\author{
V.Yu. Protasov \\ Dept. of Mechanics and Mathematics \\ Moscow State University \\ Vorobyovy Gory \\ 119992, Moscow, Russia \\ e-mail: v-protassov@yandex.ru
}

